\title{
Dual Cosmic Horizon Radius of Spacetime Curvature of a Multi-Path Connected Cosmic Topology
}

\author{
Mohammed B. Al Fadhli ${ }^{*}$ \\ ${ }^{1}$ College of Science, University of Lincoln, Lincoln, LN6 7TS, UK.
}

\begin{abstract}
The necessity of the dark energy and dark matter in the present universe could be a consequence of the antimatter elimination assumption in the early universe. In this research, I derive a new model to obtain the potential cosmic topology and the cosmic horizon radius of spacetime curvature $R_{h}(\eta)$ utilising new construal of space geometry inspired by large-angle correlations of the cosmic microwave background (CMB). I utilise a modified version of the Big Bounce theory to avoid the Big Bang singularity and inflationary constraints, and to tune the initial conditions of the curvature density. The mathematical derivation of a positively curved universe governed by only gravity revealed $\mp$ cosmic horizon solutions. Although the positive horizon is conventionally associated with the evolution of the matter universe, the negative horizon solution could imply additional evolution in the opposite direction. This possibly suggests that the matter and antimatter could be evolving in opposite directions as distinct sides of the universe, such in the visualised Sloan Digital Sky Survey. The span of the cosmic horizon radius is $R_{h}=2 \sqrt{ }\left(c^{2} t^{2}+a^{2}\right)$, which could account for the farthest observed objects. By implementing this model, we found a decelerated stage of expansion during the first $\sim 10 \mathrm{Gyr}$, which is followed by a second stage of accelerated expansion; potentially matching the tension in Hubble parameter measurements. In addition, the model predicts a final time-reversal stage of spatial contraction leading to the Big Crunch of a cyclic universe. The predicted density is $\Omega_{0}=\sim 1.14>1$. Other predictions are (1) a calculable flow rate of the matter side towards the antimatter side at the accelerated stage; conceivably explaining the dark flow observation, (2) a time-dependent spacetime curvature over horizon evolution, which could influence the galactic rotational speed; possibly explaining the high speed of stars, and (3) evolvable spacetime internal voids at the accelerated stage, which could contribute in continuously increasing the matter and antimatter densities elsewhere in both sides respectively. These findings may indicate the existence of the antimatter as a distinct side, which influences the evolution of the universe instead of the dark energy or dark matter.
\end{abstract}

Keywords: cosmology: topology, horizon, antimatter, accelerated expansion, time-reversal.

Email: malfadhli@lincoln.ac.uk; mo.fadhli7@gmail.com

\section{INTRODUCTION}

The fundamental CPT symmetry states that the matter and antimatter would have been created in the same quantities at the Big Bang [1]. In contrast, the matterantimatter asymmetry, by the violation of the CPT in the early era, could have given rise to today's matterdominated universe according to the standard Big Bang theory [2]. However, advanced measurements of the fine structure of hydrogen and antihydrogen atoms were found to be consistent with the estimations of quantum electrodynamics theory [3, 4], which contradicts the CPT violation assumption adopted in the standard Big Bang theory. Further, the monopole, horizon, and flatness problems arise from Big Bang singularity while the hypothetical theory of inflation to solve these problems was found to be problematic and unfalsifiable $[5,6]$. Moreover, current cosmological models left many unsolved problems, remarkably: the accelerated expansion of 
the universe, the fast movement of stars and the contradictory Hubble parameter measurements from the early and present universe $[12,15]$.

As an alternative, the non-singular Big Bounce theory assumes the primordial substance was concentrated from a previous collapsed universe, and the universe experiences continuous expansions and contractions [7, 8]. However, I adopt a modified version that a Bang of a thermally equilibrium primordial substance produced a hot and dense early universe where the matter and antimatter could have been separated by electromagnetic fields [9, 31]. Besides, I embrace a closed finite universe because it aids a large-scale cut-off in primaeval density fluctuations and may provide an agreement with low CMB anisotropy quadrupole observations [10].

Recently, the $\Lambda \mathrm{CDM}$ model has faced inconsistency with the advancement of new astronomical observations [12, 18]. The recent Planck Legacy 2018 (PL18) release indicated the existence of an enhanced lensing amplitude in the CMB that is higher than what is expected in the $\Lambda \mathrm{CDM}$ model $[13,14]$. This endorses the existence of a positive curvature of the universe with a level of confidence greater than 99\% [15]. In addition, the precise Hubble parameter measurements from the early universe using the Planck datasets based on the CMB show a lower value of expansion rate in comparison with the value of Hubble parameter in the present universe using the type Ia supernovae distance-redshift method [11, 19], where the variation is three standard deviations [15, 16]. Riess [19] found that the expansion of the universe is faster than what $\Lambda \mathrm{CDM}$ estimates where the disagreement between several independent measurements taken from the early and present universe is at four to six-sigma. Accordingly, a profound adjustment of the $\Lambda \mathrm{CDM}$ model or new physics are now growing due to this new evidence underlying the model assumptions [18].
In this paper, I derive a model to obtain the cosmic horizon radius while utilising a metaphorical representation based on the large-angle correlations of the CMB to help to understand the cosmic topology. I also utilise the non-singular Big Bounce theory to avoid the Big Bang singularity and to provide further tuning of the curvature density. The paper is organised as follows: In Section 2, I modify the metric tensor to account for the curvature radius of the space $R_{0}$ according to the Big Bounce theory and derive the model. In Section 3, I discuss the model implementation, matter and antimatter growth, and the model outcomes. In Section 4, I present star speed of simulation over horizon evolution, while in Section 5, I introduce the predictions of the model. In Section 6, I discuss and summarise the conclusions. Finally, in Section 7, I present future work.

\section{The Mathematical Model}

To derive the model, I utilise Einstein's field equations:

$R_{u v}-\frac{1}{2} R g_{u v}=\frac{8 \pi G}{c^{4}} T_{u v}$

where $R_{u v}$ is Ricci curvature tensor, $R$ is the scalar curvature, $g_{u v}$ is the metric tensor, $G$ is Newton's gravitational constant, $c$ is the speed of light in vacuum, and $T_{u v}$ is the energy-momentum tensor. I omitted the cosmological constant while considering gravity as the only force governing the universe [20].

The metric tensor $g_{u v}$ can be characterised using the Friedmann- Lemaître- Robertson- Walker metric (FLRW) model [23, 24]. According to the Big Bounce theory, the spatial scale factor of the universe must be greater than zero $[7,8]$. To account for initial curvature, I adjust the FLRW metric tensor $g_{u v}$ from cylindrical to spherical coordinates by incorporating dimensionless space curvature $R_{0}{ }^{2}$ where the isotropic spherical coordinates are: 


$$
\begin{aligned}
d s^{2}= & c^{2} d t^{2}-a^{2}(t)\left(\frac{d r^{2}}{1-k \frac{r^{2}}{R_{0}^{2}}}+r^{2} d \theta^{2}+\right. \\
& \left.r^{2} \sin ^{2} \theta d \phi^{2}\right)
\end{aligned}
$$

where $d s$ is the four-dimension spacetime interval in polar coordinates, $a$ is the scale factor, and $k$ is a constant representing the curvature of the space [25, 29]. The tensor signature $(+,-,-,-)$ is utilised throughout this research.

Einstein's field equations can be solved for a perfect fluid. By using the notation $c=1$ and rising one index in Equation 1, it can be expressed in term of mixed component tensors:

$R_{v}^{u}-\frac{1}{2} R \delta_{v}^{u}=8 \pi G T_{v}^{u}$

where $\delta_{v}^{u}$ is the Kronecker delta $[27,28]$. The energy-momentum tensor $T_{u v}$ of the perfect fluid is given by:

$T_{u v}=(\rho+P) u_{u} u_{v}+P g_{u v}$

where the four-dimensional velocity of the celestial fluid is $\vec{u}=(1,0,0,0), \quad u_{u}=u^{u}=$ $0, u_{t}=-u^{t}=1, \rho$ is the energy density and $P$ is the isotropic pressure [29]. By rising one index of Equation 4, it can be expressed in term of mixed component tensors:

$T_{v}^{u}=(\rho+P) u^{u} u_{v}+P \delta_{v}^{u}$

The trace tensor $T_{u}^{u}$ of the energy-momentum tensor $T_{v}^{u}$ can be obtained by contracting through indices $u$ and $v$ in Equation 3:

$R=-8 \pi G T_{u}^{u}$

where $R$ is the scalar curvature. The trace tensor of the perfect fluid is equivalent to [29]:

$T_{u}^{u} \equiv(-\rho+3 P)$
Submitting Equation 6 to Equation 3 gives:

$R_{v}^{u}=8 \pi G\left(T_{v}^{u}-\frac{1}{2} T_{u}^{u} \delta_{v}^{u}\right)$

Also, submitting Equations 5 and 7 to Equation 8 , the field equations become as follows:

$R_{v}^{u}=8 \pi G\left((\rho+P) u^{u} u_{v}+\frac{1}{2}(\rho-P) \delta_{v}^{u}\right)$

In addition, we can calculate the Ricci tensor $R_{v}^{u}$ using Christoffel symbols in Equation 10 for the adjusted metric tensor $g_{u v}$ with evoking $g_{u v} g^{v \sigma}=$ $\delta_{\sigma}^{u}$ where $\rho$ and $\sigma$ are dummy indexes and $\partial$ is a partial derivative operator:

$\Gamma_{u v}^{\rho}=\frac{1}{2} g^{\rho \sigma}\left(\partial_{u} g_{v \sigma}+\partial_{v} g_{u \sigma}-\partial_{\sigma} g_{u v}\right)$

By resolving the outcomes, we obtain the components of the Ricci tensor along the $i-i$ and $t-t$ as in Equations 11 and 12 respectively:

$R_{i}^{i}=\left(\frac{\ddot{a}}{a}+2 \frac{\dot{a}^{2}}{a^{2}}+2 \frac{k}{a^{2} R_{0}{ }^{2}}\right) \delta_{v}^{u}$

$R_{t}^{t}=3 \frac{\ddot{a}}{a}$

Submitting Equations 11 and 12 to Equation 9 while solving it along the $i-i$ and $t-t$ components give Equations 13 and 14 respectively:

$\frac{\ddot{a}}{a}+2 \frac{\dot{a}^{2}}{a^{2}}+2 \frac{k}{a^{2} R_{0}{ }^{2}}=4 \pi G(\rho-P)$

$3 \frac{\ddot{a}}{a}=8 \pi G\left(-(\rho+P)+\frac{1}{2}(\rho-P)\right)$

Combining Equations 13 and 14, we get the Friedmann equation counting the space curvature:

$\frac{\dot{a}^{2}}{a^{2}}-\frac{8 \pi G \rho}{3}+\frac{k}{a^{2} R_{0}{ }^{2}}=0$ 
Equation 15 can be solved at the time $t_{0}$ where $a_{0}$ is normalised to 1 , and $k=1$ by the definition of the metric for the positively curved universe as follows:

$d a=\sqrt{ }\left(\frac{8 \pi G}{3} \frac{\rho_{0} a_{0}{ }^{3}}{a^{3}} a^{2}-\frac{1}{R_{0}{ }^{2}}\right) d t$

where $\rho_{0} a_{0}{ }^{3}=$ constant [26].

By integrating with regards to the conformal time $d \eta \equiv \frac{d t}{a}$ in the parametric form, which is represented in the range of $(0<\eta<2 \pi)$, we can find the scale factor of the universe as follows:

$\int d \eta=\int_{0}^{2 \pi} \frac{d a}{\sqrt{\left(2 \Omega_{0, m} a-\frac{1}{R_{0}{ }^{2}} a^{2}\right)}}$

$\eta=R_{0} \sin ^{-1}\left(\frac{a}{\Omega_{0, m} R_{0}{ }^{2}}-1\right)+\frac{\pi}{2} R_{0}$

Equation 18 can be simplified where the scale factor can be written as a function of the conformal time as follows:

$a(\eta)=\frac{\Omega_{0, m} R_{0}{ }^{2}}{2}\left(1-\cos \frac{\eta}{R_{0}}\right)$

where $\Omega_{0, m}=\frac{4}{3} \pi G \rho_{0}$ is the matter density.

Also, we can obtain the universe evolution time $t(\eta)$ as a function of the conformal time using Equation 19 in term of Hubble parameter where $d t \equiv \frac{d a(\eta)}{H}[26,29]$ and integrating::

$\int d t=\int \frac{\Omega_{0, m} R_{0}^{2}}{2 H}\left(1-\cos \frac{\eta}{R_{0}}\right) d \eta$

$t(\eta)=\frac{\Omega_{0, m} R_{0}^{2}}{2 H_{0}}\left(\eta-\sin \frac{\eta}{R_{0}}\right)$

where $\frac{H^{2}}{H_{0}{ }^{2}}=\frac{\Omega_{0}}{a^{3}}+\frac{\left(1-\Omega_{0}\right)}{a^{2}}$ for a positively

curved universe and is matter-dominated [26].
The Friedmann equation of deceleration and acceleration of the universe can be obtained using Equation 14 [17]:

$\frac{\ddot{a}}{a}=-\frac{4 \pi G}{3}(\rho+3 p)$

Through inspiration by CMB large-angle correlations where the temperature correlation across the microwave sky vanishes on scalars wider than $60^{\circ}$ [30], we may metaphorically visualise the universe topology and its evolution as the evolution of a fountain; where the radius of the top circumference of the fountain (scale factor $a$ ) and its corresponding radius of curvature (horizon radius of spacetime curvature $R_{h}$ ) increase as the fountain heads away from the source (evolves in time $t$ ). Please refer to Appendix 1, Figure A.1 for the fountain schematic representation and further details.

Based on this reasoning of the non-inflationary universe, we can find the cosmic horizon radius $R_{h}(\eta)$ as a function of the conformal time as follows:

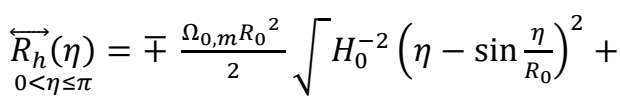

$$
\begin{aligned}
& \left(1-\cos \frac{\eta}{R_{0}}\right)^{2}, \tan ^{-1} H_{0}^{-1}(\eta- \\
& \left.\sin \frac{\eta}{R_{0}}\right)\left(1-\cos \frac{\eta}{R_{0}}\right)^{-1}
\end{aligned}
$$

The evolution of the horizon radius $\overleftrightarrow{R_{h}}(\eta)$ is in the direction of the cosmic evolution while the scale factor can be understood as a local radius. Thus, we can interpret the negative horizon radius as an indication of the existence of the antimatter as a distinct side, which is evolving in the opposite direction with regards the matter side evolution path. The span of the cosmic horizon radius is:

$R_{h}(\eta)=2 \sqrt{ }\left(c^{2} t(\eta)^{2}+a(\eta)^{2}\right)$ 
According to this new interpretation, we can differentiate $R_{h}(\eta)$ which represents the overall space curvature radius due to the overall age of the universe while $a(\eta)$ represents the universe slice radius at an instant of the time.

\section{Model Implementation and Outcomes}

\subsection{Model Implementation}

We can utilise the derived model to find the evolution of the universe over the conformal time. I assume Hubble parameter as $70 \mathrm{~km} / \mathrm{s} / \mathrm{Mpc}$ as an average of the early and today's universe, and use the approximate universe age of $\sim 10 \mathrm{Gyr}$ when the accelerated expansion started, as well as I assume $R_{0}{ }^{2}$ as 1 , thus, the model represents the exact solution of the well-known Friedmann equation.

By implementing these values, the model predicts the density is $\Omega_{0}=\sim 1.14$. In addition, the predicted evolution paths are shown in Figure 1. The model predicts that both sides of the universe are expanding in opposite directions away from the Big Bounce during the first $\sim 10$ Gyr. The slope of the evolution curves shows that the rate of expansion was slowing down, which could be due to gravity. The expansion then reached a critical horizon radius where both halves reversed their direction.

To interpret the reverse direction after passing 10 Gyr, we can utilise the metaphorical concept that the fountain reverses its direction and free-fall under gravity while its circumference continues to increase, as shown in Appendix 1, Figure A.2. Using this reasoning, we may interpret the reverse direction of the evolution paths after passing $10 \mathrm{Gyr}$ as the matter and antimatter at the critical horizon radiuses reverse their directions and free-fall towards each other due to the gravitational attraction between them at their critical horizon radiuses.
At this reverse direction stage, the expansion continues to increase as the horizon radius increases due to the evolution of the time.

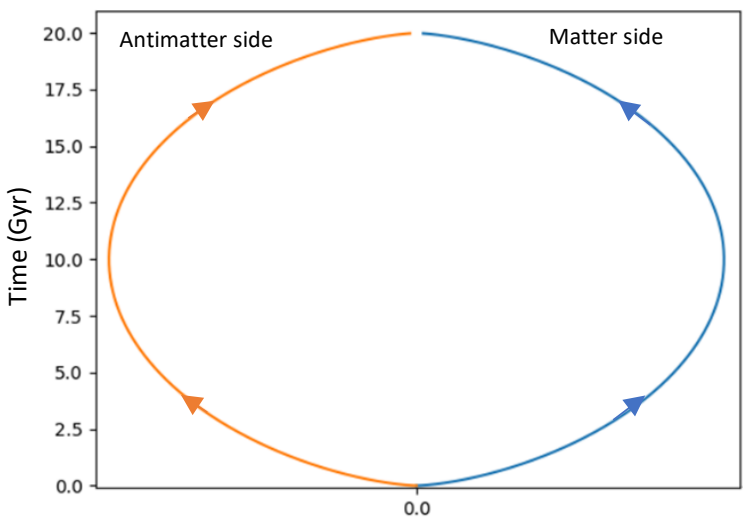

Figure 1: The evolution of the horizon radius $R_{h}$ of the matter and antimatter sides. Both sides of the universe are expanding in opposite directions away from the Big Bounce during the first 10 Gyr. Then they reverse their direction and free-fall towards each other.

Figure 2 shows a schematic representation of the expansion of both sides according to the evolution of the horizon radius.

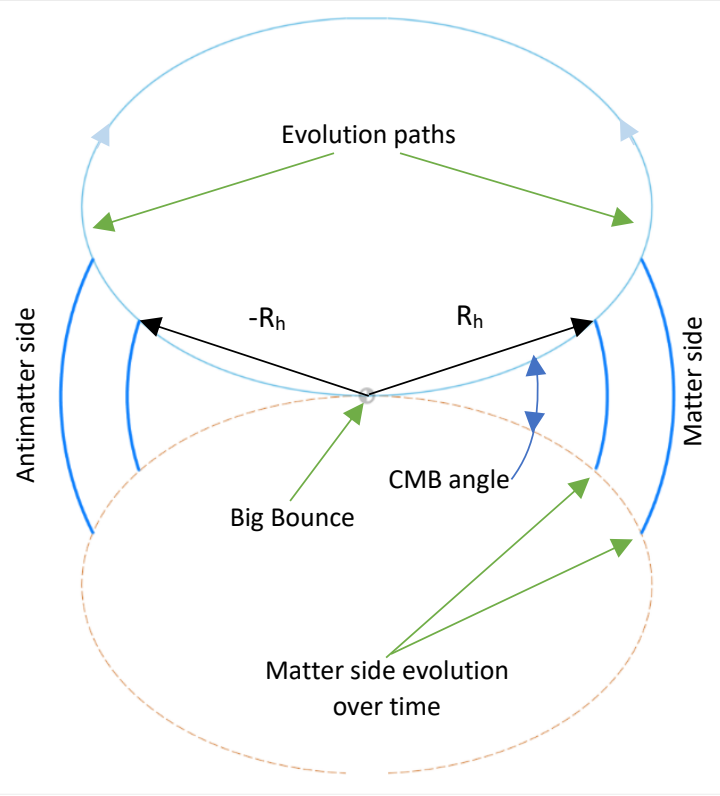

Figure 2: Schematic representation of the evolution of both sides of the universe based on the evolution of the cosmic horizon radius over the evolution in time. Both sides are expanding away from the Big Bounce during the first stage. Then they reverse their direction and free-fall towards each other. 
Nevertheless, the model should be optimised to account for the effect of the coexistence of the two sides on the matter and antimatter densities as they are approaching each other at the second stage of expansion, and to account for the possibility of spacetime void existence.

\subsection{Matter-Antimatter Density Growth}

As we interpreted that the universe is predicted to pass through two spatial stages. In the first stage, both sides spatially expand away from the Big Bounce until they reach their critical horizon radiuses. If we consider a symbolic representation of two opposite fountains, then we can expect that both matter and antimatter sides are simply path-connected at the first stage of expansion.

On the other hand, at the second stage and by considering the symbolic concept that the two opposite fountains reverse their directions and free-fall towards each other, we can expect that both sides of matter and antimatter could develop hollow spacetimes and both could be multi-path connected where spacetime voids might exist. Therefore, the spacetime voids inside the volume the universe would continuously increase the matter and antimatter densities elsewhere at both sides as the voids evolve due to the universe evolution.

Based on the Friedmann equation where $\rho a^{3}=$ constant [26]. We can mathematically reason that the matter density at the second stage $\Omega_{2, m}$ can be expressed as a function of the conformal time as follows:

$$
\begin{array}{ll}
\Omega_{2, m} \propto \eta & \pi>\eta>2 \pi \\
\Omega_{2, m}=\Omega_{c, m}\left(1+\sigma_{\Omega_{2, m}}\right) & \pi>\eta>2 \pi
\end{array}
$$

where $\Omega_{c, m}$ is the critical matter density and $\sigma_{\Omega_{2, m}}$ is assumed as the matter density growth factor.

In addition, based on the Friedmann equation of the deceleration and acceleration, the expansion rate is a function of the scale factor while the scale factor is a function of the conformal time. Therefore, we can account for the variable expansion rates where the Hubble parameter can be considered as a second-order function of the conformal time for both stages of expansion as follows:

$H_{1}=H_{i}\left(1+\sigma_{H_{1}}\right)^{-2} \quad 0>\eta>\pi$

$H_{2}=H_{c}\left(1+\sigma_{H_{2}}\right)^{2} \quad \pi>\eta>2 \pi$

where $H_{i}, H_{1}, H_{c}, H_{2}$ represent the Hubble parameter at the initial, first, critical, and second stages respectively; $\sigma_{H_{1}}$ is assumed as the Hubble decrease factor at the first stage and $\sigma_{H_{2}}$ is assumed as the Hubble increase factor at the second stage.

Accordingly, the optimised model of the universe during the first and second stages of expansion is as follows respectively:

$$
\begin{aligned}
\underset{0<\eta \leq \pi}{\overleftrightarrow{R_{h}}}(\eta)=\mp & \frac{\Omega_{0, m} R_{0}{ }^{2}}{2} \sqrt{ } H_{1}^{-2}\left(\eta-\sin \frac{\eta}{R_{0}}\right)^{2}+ \\
& \left(1-\cos \frac{\eta}{R_{0}}\right)^{2}, \tan ^{-1} H_{1}^{-1}(\eta- \\
& \left.\sin \frac{\eta}{R_{0}}\right)\left(1-\cos \frac{\eta}{R_{0}}\right)^{-1}
\end{aligned}
$$

$$
\begin{aligned}
\underset{\pi<\eta \leq 2 \pi}{\overleftrightarrow{R_{h}}}(\eta)=\mp & \frac{\Omega_{2, m} R_{0}{ }^{2}}{2} \sqrt{ } H_{2}^{-2}\left(\eta-\sin \frac{\eta}{R_{0}}\right)^{2}+ \\
& \left(1-\cos \frac{\eta}{R_{0}}\right)^{2}, \tan ^{-1} H_{2}^{-1}(\eta- \\
& \left.\sin \frac{\eta}{R_{0}}\right)\left(1-\cos \frac{\eta}{R_{0}}\right)^{-1}
\end{aligned}
$$




\subsection{Optimised Model Outcomes}

Both Hubble parameter measurements at the early universe of $\sim 67.4 \mathrm{~km} / \mathrm{s} / \mathrm{Mpc}$ and the current universe of $\sim 73.5 \mathrm{~km} / \mathrm{s} / \mathrm{Mpc}$ at universe ages of $\sim 0.37$ and $\sim 13.8 \mathrm{Gyr}$ respectively as well as the approximate age of $\sim 10$ Gyr when the accelerated expansion started were used in tuning the optimised model. Due to the symmetry of both sides, we can investigate the evolution of the matter side while the antimatter side should evolve similarly but in the opposite side. Three cases with different values of the model parameters as illustrated in Table 1 were investigated:

Table 1: Model parameters

\begin{tabular}{|l|l|l|l|l|l|}
\hline & $\Omega_{0, m}$ & $R_{0}{ }^{2}$ & $\sigma_{\Omega_{e, 2}} \%$ & $\sigma_{H_{1}} \%$ & $\sigma_{H_{2}} \%$ \\
\hline Case 1 & 1.14 & 1 & 0.001 & 0.01 & 0.07 \\
\hline Case 2 & 1.14 & 1.1 & 0.001 & 0.01 & 0.07 \\
\hline Case 3 & 1.16 & 1.1 & 0.001 & 0.01 & 0.07 \\
\hline
\end{tabular}

By implementing these parameters, we obtain the predicted evolution paths of the horizon radius as shown in Figure 3. The model predicts that cosmic evolution might experience three distinct stages. Firstly, the matter half of the universe was expanding away from the Big Bounce during first 10 Gyr with a decelerated spatial expansion, which could be due to gravity between the two sides, until it reaches its critical horizon radius.

Secondly, after passing $\sim 10 \mathrm{Gyr}$, an accelerated stage of a reverse direction of the universe expansion started, which could be due to the gravitational attraction between the matter and antimatter at their critical horizon radiuses. The matter and antimatter could be under free-fall towards each other at gravitational acceleration, causing the current accelerated expansion of the universe.

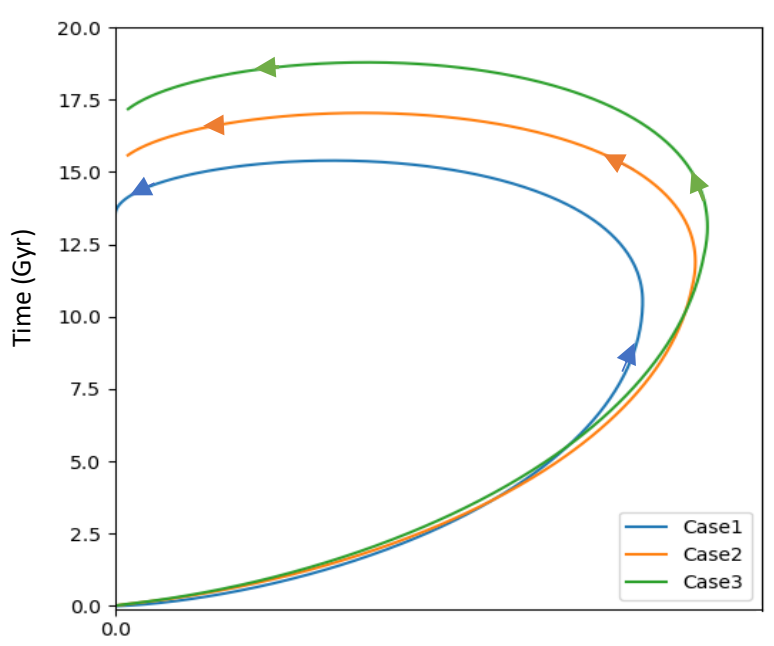

Figure 3: Evolution of the horizon radius $R_{h}$ of the matter side. Firstly, a decelerated stage of spatial expansion away from the Big Bounce until it reaches its critical radius. Secondly, an accelerated stage of a reverse direction. Finally, a time-reversal stage of spatial contraction.

Interestingly, the model predicts a third stage of spatial contraction with reversal-time direction at $\sim 16.5 \mathrm{Gyr}$ and afterwards (depends on the tuning of the model conditions). At this stage, the universe experiences a stage of contraction which could lead to final collapse in the Big Crunch. Based on the evolution paths of the horizon radius, we can schematically represent the predicted cosmic topology as shown in Figures 4.

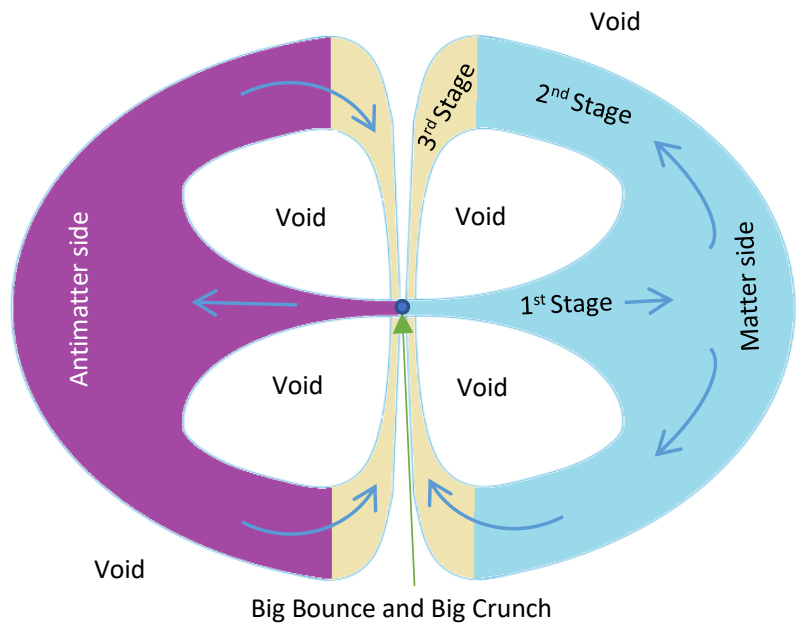

Figure 4: 2D-Schematic representation of the predicted actual cosmic topology of both sides. Internal voids are predicted to form at the second stage of reverse direction. 
However, due to gravitational lensing and how we observe light, the universe during the first and second (current) stages would appear as shown in Figure 5, potentially matching the Sloan Digital Sky Survey Data. Please refer to Appendix 1, Figure A.3 for 3-D visualization of Sloan Digital Sky.

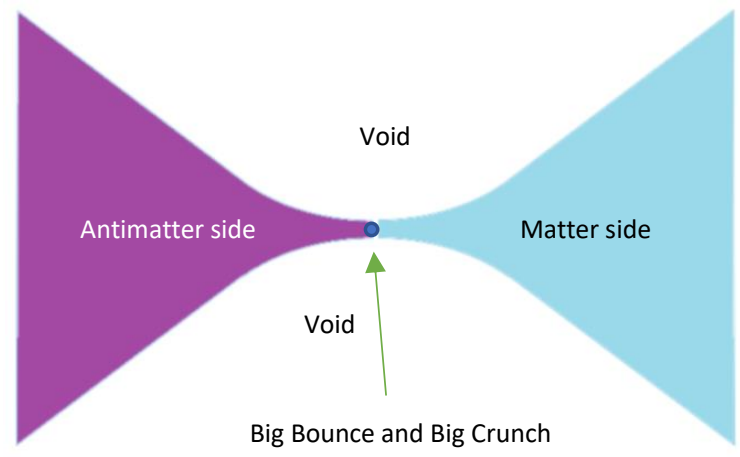

Figure 5: 2D-Schematic representation of the apparent topology, due to gravitational lensing, of the universe during the first and second (current) stages of expansions.

\section{N-body Simulations of Galaxy Rotation}

The consistent patterns of galactic rotation curves using precise and independent galactic redshift data confirmed that the hydrogen clouds and outer stars are orbiting galaxies at speeds faster than that calculated using Newtonian laws. Accordingly, the dark matter hypothesis was introduced to account for the apparently missing galactic mass and to explain the fast-orbital velocity [32, 33]. However, no evidence of the existence of the dark matter, which is supposed to account for the majority galactic mass, was observed since its introduction. The failure to find dark matter led to the introduction of new theories such as modified gravity and modified Newtonian dynamics [21, 22, 34 - 36]. On the other hand, several recent studies found that many galaxies do not contain dark matter [37 - 39]. This observation was considered in some studies where the galaxy formation was simulated using modified Newtonian dynamics without considering the dark matter [40]. Thus, it seems that there is no evidence or agreement on the existence or nature of the dark matter as well as it is not an essential element in some galaxies.

As an alternative, I introduce a new hypothesis based on the variation of the curvature of both sides along with the evolution of the horizon radius as was shown Figure 4, where the curvature of the spacetime varies at different instant of the time. Accordingly, I argue that the observed various star movements could occur as a result of the variation of the universe curvature, where the gravitational attraction between both sides of the universe can highly bend the spacetime over the evolution of the horizon radius.

To evaluate this hypothesis, I perform a fluid simulation study based on the Newtonian dynamics using the Fluid - Pressure and Flow software [41]. In this simulation, a perfect fluid of mass density $\rho$ and isotropic pressure $p$ was assumed to represent the matter of the universe while the fluid particles were assumed to represent the stars. The fluid was considered as a perfect fluid because it is frictionless with no heat conductivity [42]. Using these conditions, the fluid model was built to simulate the star movement speed between an incrementing horizon curvature and their distance from it to a lesser curved spacetime, as shown in Figure 6.

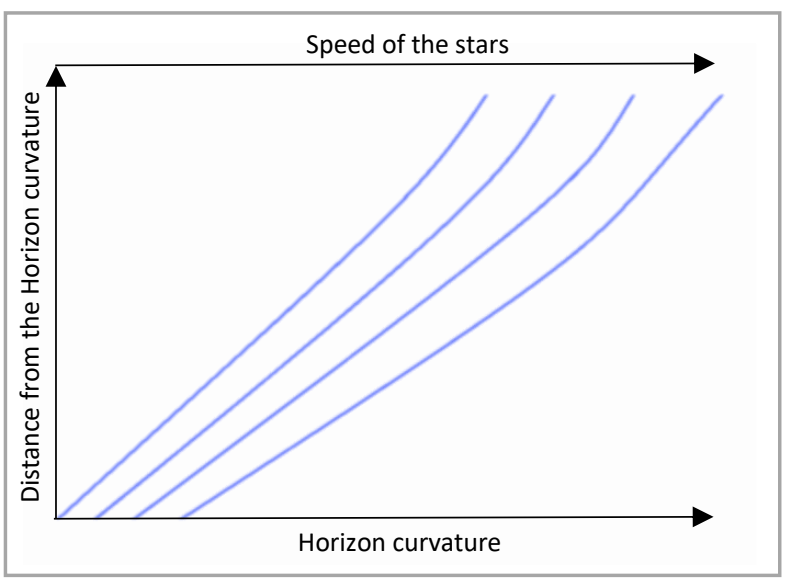

Figure 6: The particles that passing away from the highly curved horizon are moving faster than their counterparts that are passing within highly curved spacetime. As the bending of horizon increases, the speed difference increases. 
Considering these outcomes, it could be concluded that the variation of the curvature of the spacetime over the horizon evolution can influence the speed of star movement as they are located through different curvatures of the universe.

\section{The Predictions of the Model}

This model predicts the rate of expansion varies over time. The expansion rates at the first and second stage of spatial expansion can be estimated according to the Friedmann equation (Equation 22). Accordingly, we can find the normalised deceleration and acceleration of the universe over the conformal time for the stated three cases in Section 3.3, as shown in Figure 7.

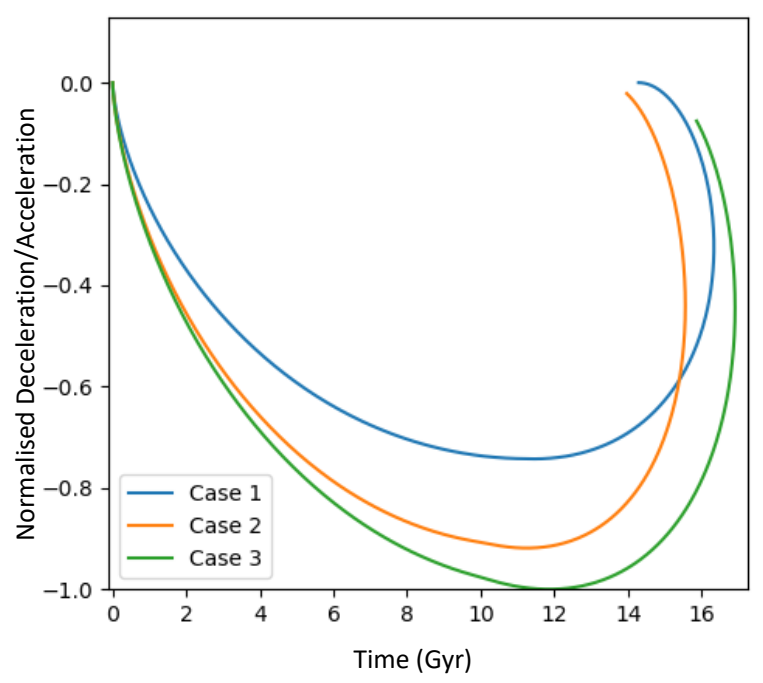

Figure 7: The deceleration and acceleration of the universe expansion rate over time.

The expansion rate was decreasing at the first stage, and this conforms with the lower value of the Hubble parameter obtained by the Planck datasets of the early universe $[12,15]$. However, at the second stage, the expansion rate is increasing, and this aligns with the higher value of the Hubble parameter obtained using the supernovae type Ia distance-redshift method at the present universe.

In this dual-side universe, each side is not a mirror image of the other side, but each is a separate identity where galaxies can evolve and spread in different locations and shapes. A new 'Cosmic Conservation' term can be suggested to illustrate that this finite and positively curved universe has a fixed amount of energy [7, 8]. Therefore, the Cosmic Conservation can explain the instantaneous quantum entanglement where the finite universe conserves the total spin of a pair of particles regardless of their locations. Therefore, we can predict that the locations of quantumly entangled particles do not affect their entanglement as their total spin is conserved. Otherwise, the universe is not finite.

At the second stage of expansion, the model predicts a flow of the matter side of the universe due to the gravitational attraction by the antimatter side. We can predict the flow rate using the derivative of the optimised matter model with regards to the conformal time as follows:

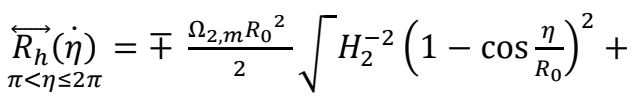

$$
\begin{aligned}
& \left(\sin \frac{\eta}{R_{0}}\right)^{2}, \tan ^{-1} H_{2}^{-1}(1- \\
& \left.\cos \frac{\eta}{R_{0}}\right)\left(\sin \frac{\eta}{R_{0}}\right)^{-1}
\end{aligned}
$$

Finally, we can avoid the black hole singularity by considering the possibility that the black holes in the matter side could be tunnelled to antimatter white holes in the other side of the universe through Einstein-Rosen bridge or wormhole [43]; where physical information can be quantumly tunnelled to the antimatter white holes and never being lost. The information would change its charge as it passes to the antimatter side which could have an opposite CPT time arrow in agreement with CPT symmetry and according to the interpretation of Stueckelberg [44] that the antimatter travels backwards in time with regards to the travel direction of the matter. 


\section{Conclusions}

In this research, I derived a new model of dual horizon radius of space curvature. The model recommends the universe consists of two sides of matter and antimatter that are symmetrical in geometry and evolve in opposite directions. I used the model to simulate the evolution of the horizon radius of the universe. The results predicted that the expansion rate is variable. The rate during the early universe was decreasing while the rate at the current universe is increasing. These results conceivably align with Hubble measurements from the early and current universe observations. The results also indicate that the universe would experience a third stage of accelerated contraction towards the Big Crunch in the reverse time direction. The model also predicts a flow rate of the matter side towards the antimatter side at the accelerated stage of expansion.

Regarding the movement of the star, the fluid simulation can provide a plausible explanation where the highly curved spacetime fabric of both matter and antimatter sides could drive stars located at a lesser curved spacetime to move faster than their counterparts that are located within a highly curved fabric.

The abandonment of the cosmological constant in this model could fit the quantum field theory as it distinguishes the quantum vacuum energy from the energy of space and attributes the accelerated expansion to the gravitational attraction between both sides. However, the literature on dark matter/energy can be utilised to accurately estimate the matter density distribution and its growth factor.

\section{Future Work}

The values of the matter density, curvature density, energy growth factor, the Hubble parameter change factors at both stages have act a vital role in the estimation of the universe evolution paths. Therefore, accurate values can provide a better estimation of the evolution paths. The possibility that the black holes in the matter side could be tunnelled to antimatter white holes in the other side of the universe through Einstein-Rosen bridge or wormhole can be investigated to avoid the black hole singularity. Finally, the accurate age of the universe can be estimated based on the predicted non-linear Hubble parameter.

\section{References}

[1] Mavromatos, N.E., 2017. Models and (some) searches for CPT violation: from early universe to the present era. In Journal of Physics: Conference Series (Vol. 873, No. 1, p. 012006). IOP Publishing.

[2] Andrei Sakharov. 1967. Violation of CP symmetry, C-asymmetry and baryon asymmetry of the universe: Vol.5 JETP Letter, pp. 24-27

[3] Eriksson S. 2018. Precision measurements on trapped antihydrogen in the ALPHA experiment. Philosophical transactions. Series A, Mathematical, physical, and engineering sciences, 376(2116), 20170268. https://doi.org/10.1098/rsta.2017.0268

[4] Ahmadi, M., Alves, B.X.R., Baker, C.J. et al. 2020. Investigation of the fine structure of antihydrogen. Nature 578, 375-380.

[5] Gonzalo, J.A., 2005. Inflationary Cosmology Revisited: An Overview of Contemporary Scientific Cosmology After the Inflationary Proposal. World Scientific.

[6] Steinhardt, Paul J. (2011). "The inflation debate: Is the theory at the heart of modern cosmology deeply flawed?". Scientific American. 304 (4): 18-25. doi:10.1038/scientificamerican0411-36 
[7] Trautman, A. 1973. Nature (Phys. Sci.) 242, 7.

[8] Unger, G. and Popławski, N., 2019. Big bounce and closed universe from spin and torsion. The Astrophysical Journal.

[9] O. Klein, "Instead of Cosmology“, PEER REVIEWED Nature, 211, 1337 - 1341 (1966)

[10] Efstathiou, G. 2003. Is the low cosmic microwave background quadrupole a signature of spatial curvature? Mon. Not. Roy. Astron. Soc. 343, L95-L98

[11] Riess, A.G., Filippenko, A.V., Challis, P., Clocchiatti, A., Diercks, A., Garnavich, P.M., Gilliland, R.L., Hogan, C.J., Jha, S., Kirshner, R.P. and Leibundgut, B.R.U.N.O., 1998. Observational evidence from supernovae for an accelerating universe and a cosmological constant. The Astronomical Journal, 116(3), p.1009.

[12] E. Di Valentino, A. Melchiorri and J. Silk. 2020. Cosmic Discordance: Planck and luminosity distance data exclude LCDM. arXiv preprint arXiv:2003.04935.

[13] Aghanim, N. et al. 2019a (Planck Collaboration) Planck 2018 results. VI. Cosmological parameters.

[14] Aghanim, N. et al. 2019b (Planck Collaboration) Planck 2018 results. V. CMB power spectra and likelihoods.

[15] E. Di Valentino, A. Melchiorri and J. Silk. 2019. Planck evidence for a closed Universe and a possible crisis for cosmology.

[16] Riess, A. G. et al. 2018. New parallaxes of galactic cepheids from spatially scanning the Hubble Space Telescope: implications for the Hubble constant. Astrophys. J. 855, 136.

[17] Friedmann, A. 1922. Z. Phys. 10, 377.

[18] Lusso, E., Piedipalumbo, E., Risaliti, G., Paolillo, M., Bisogni, S., Nardini, E. and Amati, L., 2019. Tension with the flat Lambda CDM model from a high redshift Hubble Diagram of supernovae, quasars and gammaray bursts. arXiv preprint arXiv:1907.07692.

[19] Riess, A.G., 2020. The expansion of the Universe is faster than expected. Nature Reviews Physics, 2(1), pp.10-12.

[20] Einstein, Albert.1916. "The Foundation of the General Theory of Relativity". Annalen der Physik. 354 (7): 769.

[21] Maeder, Andre. 2017. An Alternative to the $\Lambda$ CDM Model: The Case of Scale Invariance". The Astrophysical Journal. 834 (2):194. arXiv:1701.03964. Bibcode:2017ApJ...834..194M. doi:10.3847/15384357/834/2/194. ISSN 0004-637X.

[22] Brouer, Margot. 2017. "First test of Verlinde's theory of emergent gravity using weak gravitational lensing measurements". Monthly Notices of the Royal Astronomical Society. 466 (3): 25472559. arXiv:1612.03034. Bibcode:2017MNRAS.466.2547B. doi:10.1093/mnras/stw3192.

[23] Lachieze-Rey, M.; Luminet, J.-P. 1995. "Cosmic Topology", Physics Reports, 254 (3): 135-214, arXiv:grqc/9605010, Bibcode:1995PhR...254..135L, doi:10.1016/0370-1573(94)00085-H

[24] Ellis, G. F. R.; Elst, H. van. 1999. "Cosmological models (Cargèse lectures 1998)". In Marc Lachièze-Rey (ed.). Theoretical and Observational Cosmology. NATO Science Series C. 541. pp. 1-116. arXiv:grqc/9812046.

[25] Landau, L. D.; Lifshitz, E. M. 1975. The Classical Theory of Fields (Pergamon).

[26] Ryden, B. 2003. Introduction to cosmology. Introduction to cosmology / Barbara Ryden. San Francisco, CA, USA: Addison Wesley, ISBN 0-8053-8912-1.

[27] Carmeli, Moshe. 1982. Theory and practice Classical Fields: General Relativity and Gauge Theory New York: John Wiley xvii+650 ISBN 0471864374

[28] Carmeli, Moshe. 2001. Classical fields: general relativity and gauge theory. World Scientific Publishing Company.

[29] Norbert, Straumann. 2013. General Relativity, Graduated Texts in Physics, Second Edition, Springer.

[30] Luminet, J., Weeks, J., Riazuelo, A. et al. Dodecahedral space topology as an explanation for weak wideangle temperature correlations in the cosmic microwave background. Nature 425, 593-595 (2003). https://doi.org/10.1038/nature01944

[31] Oskar Klein, “Arguments concerning Relativity and Cosmology” PEER REVIEWED Science 29 January 1971: Vol. 171. no. 3969, pp. 339 - 345

[32] Mannheim, P.D. and Kazanas, D., 1989. Exact vacuum solution to conformal Weyl gravity and galactic rotation curves. The Astrophysical Journal, 342, pp.635-638. 
[33] Yoshiaki Sofue, Vera Rubin. 2000. Rotation Curves of Spiral Galaxies, arXiv. https://arxiv.org/pdf/astroph/0010594.pd

[34] Edmund A. Chadwick, Timothy F. Hodgkinson, and Graham S. McDonald. 2013. Gravitational theoretical development supporting MOND, Phys Rev D Phys. Rev. D 88, 024036

[35] J.R. van Meter. 2018. Dark-matter-like solutions to Einstein's unified field equations, Phys. Rev. D 97 , 044018.

[36] Mordehai Milgrom. 2019. MOND in galaxy groups: A superior sample, Phys Rev D 99, 044041

[37] Dodelson, S. and Liguori, M., 2006. Can cosmic structure form without dark matter?. Physical Review Letters, 97(23), p.231301.

[38] Guo, Q., Hu, H., Zheng, Z., Liao, S., Du, W., Mao, S., Jiang, L., Wang, J., Peng, Y., Gao, L. and Wang, J., 2019a. A population of dwarf galaxies deficient in dark matter. arXiv preprint arXiv:1908.00046.

[39] Guo, Q., Hu, H., Zheng, Z., Liao, S., Du, W., Mao, S., Jiang, L., Wang, J., Peng, Y., Gao, L. and Wang, J., 2019b. Further evidence for a population of dark-matter-deficient dwarf galaxies. Nature Astronomy, pp.16.

[41] Sam Reid et al. 2013. Fluid Pressure and Flow, PhET Interactive Simulations. University of Colorado

[40] Wittenburg, N., Kroupa, P. and Famaey, B., 2020. The formation of exponential disk galaxies in MOND. arXiv preprint arXiv:2002.01941.

[42] Nemiroff, R.J.; Patla, B., 2008. Adventures in Friedmann cosmology: A detailed expansion of the cosmological Friedmann equations. American Journal of Physics.

[43] Weyl, H. 1921. "Feld und Materie". Annalen der Physik. 65 (14): 541-563. Bibcode:1921AnP...370..541W. doi:10.1002/andp.19213701405.

[44] Stueckelberg, E.C.G. 1941. Helvetica Physica Acta, 14, 588-594.

[45] SLOAN Digital Sky Survey (3-D Visualization of Visible Universe). Online link: https://www.youtube.com/watch?v=_pDZW-RAXcc 


\section{Appendix 1: Fountain Representation}

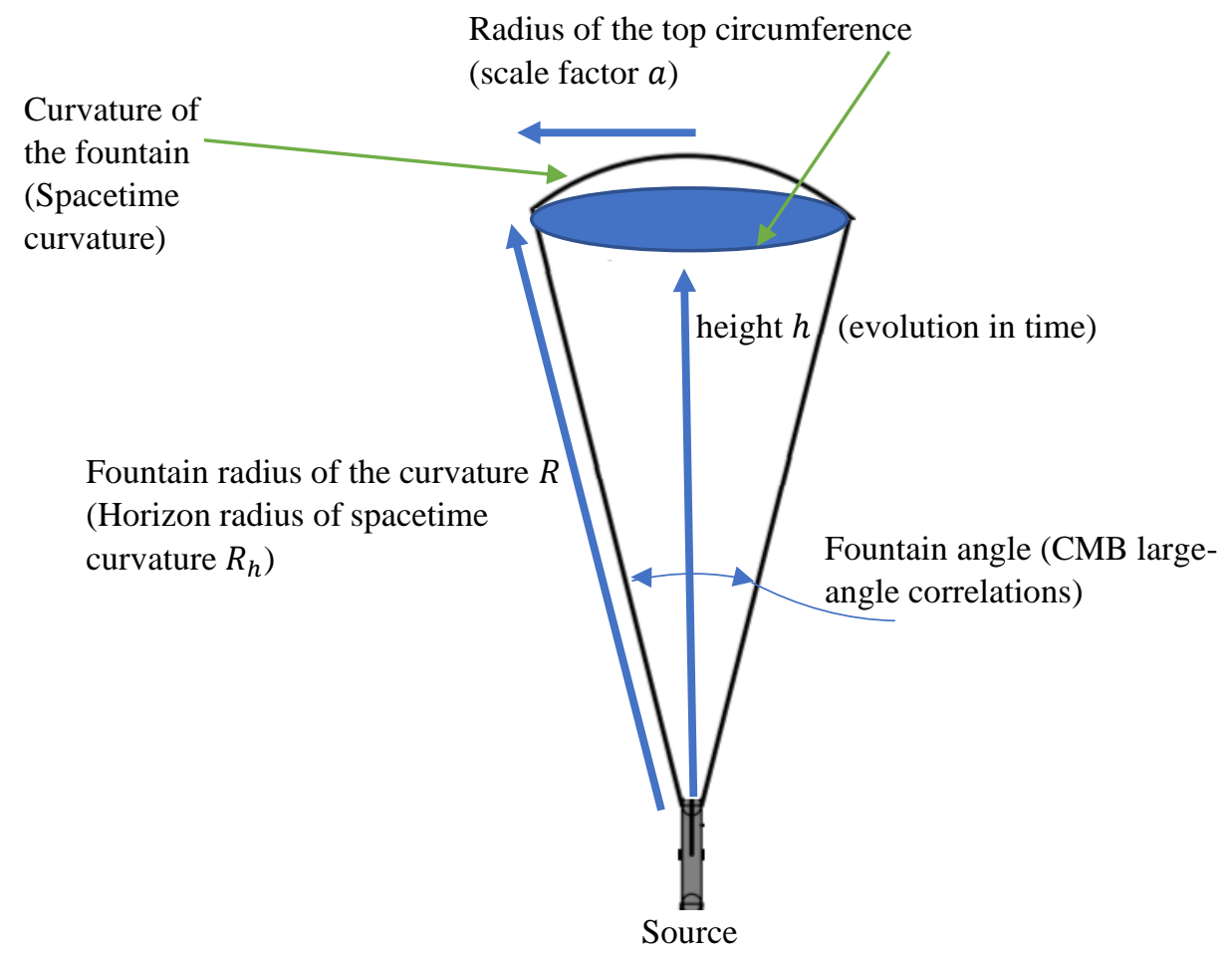

Figure A.1: The fountain representation. Both the height of the fountain and its radius of the curvature are evolving in time upwards while its radius of the top circumference represents its size at an instant of the time.

According to Pythagorean theorem, the radius of curvature of the fountain $R$ can be calculated as follows:

$R^{2}=h^{2}+a^{2}$

Taking square root for both sides:

$R=\mp \sqrt{ }\left(h^{2}+a^{2}\right)$

where the height can be found as the speed of the water multiply by the time

If we apply this concept on the spacetime of the universe where $c t$ replaced the height of the fountain, we get:

$R_{h}^{2}=c^{2} t^{2}+a^{2}$

$R_{h}=\mp \sqrt{ }\left(c^{2} t^{2}+a^{2}\right)$ 


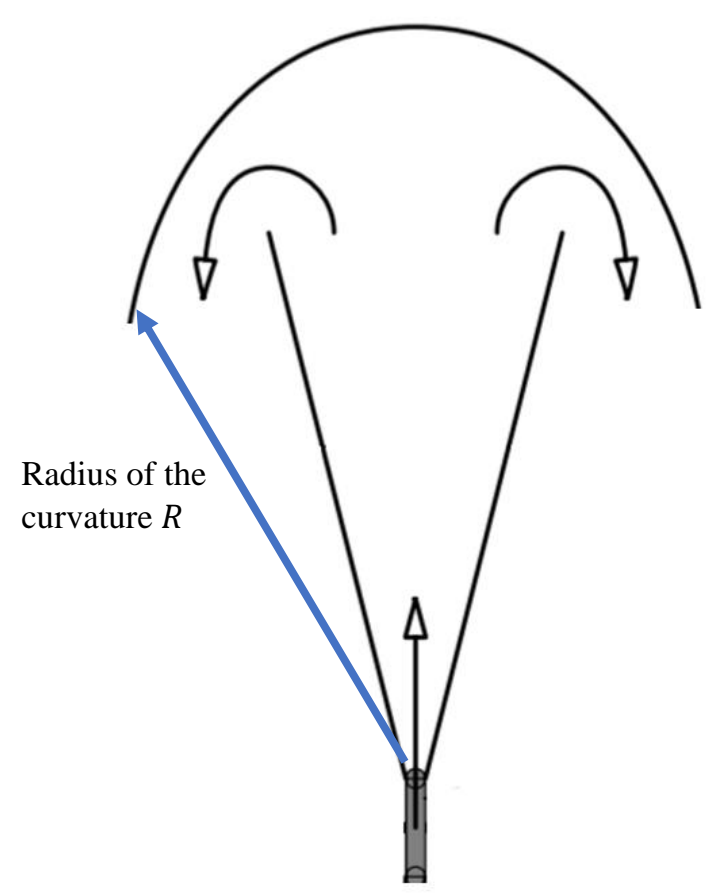

Figure A.2: Fountain Representation of upwards and downwards directions.

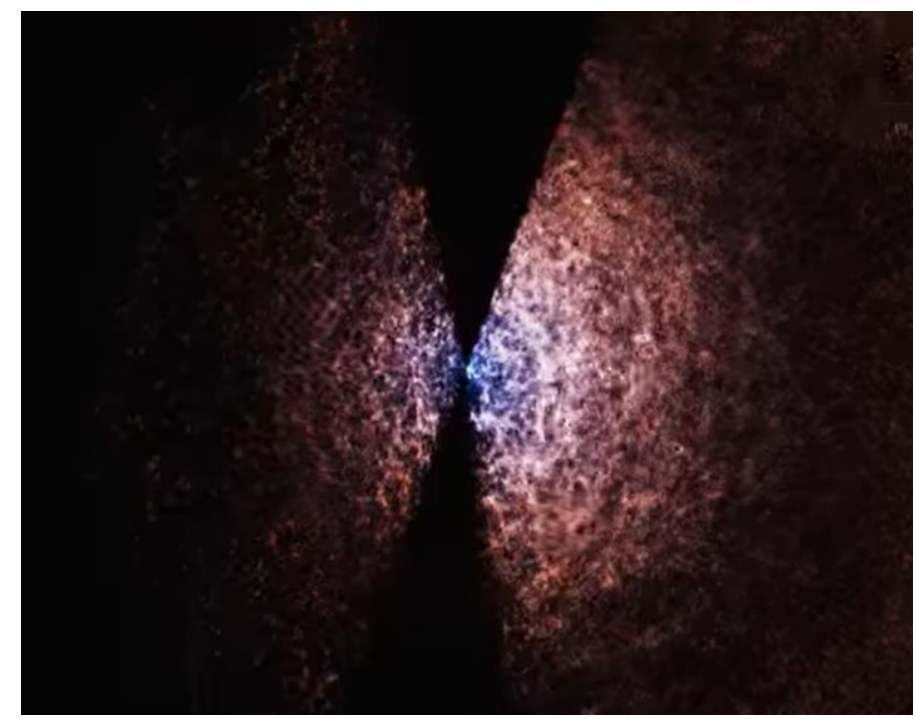

Figure A.3: SLOAN Digital Sky Survey (3-D Visualization of Visible Universe) [45]. 